\title{
DISCIPLINAMIENTO DEL CUERPO DE LAS MUJERES ${ }^{1}$
}

\section{DISCIPLINING WOMEN’S BODIES}

\section{Blanca Luz Sojo Mora*}

\section{RESUMEN}

Es este artículo se busca determinar las acciones que realizan las mujeres para controlar y moldear su cuerpo, así como, las razones por las que recurren al disciplinamiento corporal. Para ello se realizó una entrevista a 14 participantes. Se encontró que las mujeres entrevistadas recurren a varias acciones para disciplinar su cuerpo, siendo la actividad física la principal; además, se encontró que ese disciplinamiento está relacionado con la insatisfacción corporal, la preocupación por la apariencia y con las normas de género. En conclusión, las mujeres disciplinan sus cuerpos para tener una "apariencia femenina y atractiva", según los ideales estéticos sociales.

\section{PALABRAS CLAVE: MUJERES * DISCIPLINA * CUERPO * FEMINIDAD * PODER}

\section{ABSTRACT}

This article determines the actions that women perform to control their body, as well as the reasons why they use corporal discipline. In this study, an interview was conducted with 14 participants. It was found that the women interviewed perform various actions to discipline their body, the main one being physical activity. It was also found that this discipline is related to body dissatisfaction, concern for appearance and gender norms. In conclusion, women discipline their bodies to have a "feminine and attractive appearance", according to social aesthetic ideals.

KEYWORDS: WOMEN * DISCIPLINE * BODY * FEMININITY * POWER

1 Este artículo de investigación responde a la Tesis "Construcción de las subjetividades femeninas por medio de la práctica de Actividades Físico-Deportivas" desarrollada para optar por el grado de Doctorado en Estudios de la Sociedad y la Cultura, presentado en el año 2015. 
1) MARCO REFERENCIAL

\section{EL CUERPO}

En los últimos tiempos, se ha publicado literatura en relación con el cuerpo, desde diferentes temas y perspectivas. Sin embargo, es con el surgimiento de la fenomenología, corriente filosófica del siglo xx que inicia el interés por el estudio del cuerpo. Pero, ¿qué es el cuerpo? Bordo (1992) cita a Bourdieu y a Foucault para decir que el cuerpo es un centro de control social; es a través de maneras, hábitos, rutinas, reglas y prácticas que la cultura construye el cuerpo (Bordo,1992).

Por otro lado, Lagarde (1996) afirma que "el cuerpo es el más preciado objeto de poder" (p. 56). Indica que son las instituciones quienes disciplinan, controlan y recrean los cuerpos a través de diversos procesos que enseñan cómo debe ser el cuerpo para hombres o para mujeres, según las normas corporales de género, por lo tanto, cada cuerpo es disciplinado con fines sociales.

Otra autora que se refiere a la construcción corporal es Butler (2007), quien afirma que el cuerpo es un instrumento relacionado externamente con un conjunto de significados culturales, por lo tanto, es una construcción cultural.

Martínez (2001) también vincula el cuerpo con la cultura, detalla que "sobre el cuerpo recae la significación que le otorga la cultura” (p. 35). El significado que adquiere el cuerpo depende de la cultura, usos, valores, creencias, cánones estéticos, incluso, las clases sociales influyen en la diversificación de sus significados.

También Gatens (2002) menciona que la diferencia entre los cuerpos de hombre 0 mujer no se relaciona con lo biológico, sino con "la forma en que la cultura marca los cuerpos $y$ crea condiciones específicas en que pueden vivir y recrearse" (p. 146).

En esta investigación, el concepto de cuerpo que se maneja, más que biológico es social, en el que la cultura le otorga un significado. Entendido el cuerpo, según las citas anteriores, como un centro de control social sobre el que se ejerce poder con el fin de disciplinarlo.

\section{EL PODER Y EL DISCIPLINAMIENTO}

Al tratar el disciplinamiento corporal, resulta necesario introducir el tema del poder, porque según la literatura, el poder se ejerce sobre el cuerpo. Según Caamaño y Rangel (2002) “...el poder proviene desde abajo; está inducido en el cuerpo y producido en cada interacción social" (p. 81), y confirman que "el cuerpo se vuelve un locus de control privilegiado, el lugar de dominio a través del cual se pretende lograr la docilidad y constituir la subjetividad" (p. 81). En esta misma línea temática, Bourdieu (2000) explica cómo el poder se ejerce directamente sobre los cuerpos "como arte de magia al margen de cualquier coacción física" (p. 54).

Por otra parte, para Foucault (2008) el poder no es una institución, dominación de un grupo sobre otro o control ejercido por oposición, sino una serie de relaciones, mecanismos $y$ estrategias que prescinden de la violencia. Considera que el cuerpo está inmerso en relaciones de poder y que el sometimiento del cuerpo no se obtiene solo mediante la violencia, sino que puede ser muy sutil; esto para lograr cuerpos dóciles, es decir, cuerpos que puedan ser sometidos, utilizados, transformados y perfeccionados.

En cuanto al poder como una multiplicidad de relaciones que se reproducen en todas partes y en toda relación, Foucault (1979) explica que "entre cada punto del cuerpo social, entre un hombre y una mujer, en una familia, entre un maestro y su alumno, entre el que sabe $y$ el que no sabe, pasan relaciones de poder" (p. 157).

Foucault (2008) explica que ese poder funciona "mediante el juego de las miradas... miradas que deben ver sin ser vistas" (p. 200); es así como las miradas permiten un control y vigilancia constante. En esta misma línea, Andrews, Cole y Giardina (2004) mencionan que, según las teorías de Foucault, el poder moderno funciona invisiblemente pero sus 
efectos son visibles, e indican que Foucault lo ejemplifica con el panóptico ${ }^{2}$ de Bentham.

En su primera carta, Bentham (2011) indica que "a lo que se debe aspirar es a que en todo momento el recluso tenga razones para creerse vigilado" (p. 40). De la misma manera en que "los presos" interiorizan la mirada de quien los vigila hasta vigilarse a sí mismos, las mujeres y los hombres vigilan sus cuerpos.

Lee (1997) cita el modelo panóptico dentro de la sociedad disciplinaria en el que cada persona es su propio carcelario de manera consciente; explica que esto es una señal de control del cuerpo y una característica de la época actual, que se orienta al aumento de la conciencia de sí.

Por su parte, Martínez (2001) se basa en las argumentaciones de Foucault para afirmar que existe una "estrategia de normalización, que busca la producción de cuerpos dóciles, capaces de auto-control y de autodisciplina dispuestos a transformarse $y$ a mejorarse al servicio de las normas sociales" (p. 55). Argumenta que el poder no solo usa la violencia física, sino que solamente basta con una mirada para que cada individuo se controle a sí mismo. De esta manera, las disciplinas de la dieta y el ejercicio surgen como prácticas normativas de la feminidad, pero para las mujeres se experimentan esas "prácticas como fuentes de poder y de control en tanto las perciben como medios para alcanzar la belleza, la aceptación social, laboral y sexual, en suma la posibilidad de influir en los otros" (p. 55). Esta misma autora expresa que "se debe tener la sensación duradera y permanente que somos dueños de nuestro cuerpo para poder dirigirlo donde deseamos sin interferencias" (Martínez, 2001, p. 143).

Por ello, se entiende el cuerpo como centro de poder $y$ de control, pero en este caso, de una forma difícil de detectar, una manera

2 El concepto panóptico se refiere a una arquitectura carcelaria que permite al guardia observar a todas las personas prisioneras sin que ellas puedan saber si son observadas. Es considerado un dispositivo moderno de vigilancia que trasciende la prisión y llega a las escuelas, fábricas, hospitales, entre otros, con el fin de imponer una conducta mientras se mira a los otros sin ser vistos. silenciosa e invisible en la que no se recurre a la violencia física, sino a una estrategia de autocontrol corporal. Según las citas anteriores, se está bajo las miradas externas, las cuales incitan a prácticas como la dieta y el ejercicio, con el fin de responder a un ideal de corporeidad.

Es necesario agregar que la feminidad es una construcción cultural impuesta como ideal social para las mujeres, representado con gestos, vestimenta y comportamiento. Esta condición asignada a las mujeres responde a lo visible ante las demás personas. González (1999) lo explica al afirmar que lo femenino y lo masculino "se trata de creaciones culturales que se ofrecen (o se imponen) a los sujetos como modelos ideales que, a su vez, se incorporan a los individuos bajo la forma de un ideal del yo" (p. 61).

\section{2) ANTECEDENTES}

Se encontraron varias investigaciones con hallazgos relevantes en torno a este tema. Sin embargo, es necesario aclarar que en la mayoría de estos estudios, la metodología no es equivalente a la utilizada en esta investigación, esto es una limitante al ser usadas comparativamente.

Por su parte, Sossa (2011) retoma a Foucault para explicar cómo funcionan los fenómenos de consumo que promueven la delgadez y la juventud, los cuales condicionan un estado de salud, belleza y felicidad. Entre las conclusiones de su ensayo, se refiere a la belleza física y al consumo, enfatizando el poder que se ejerce para clasificar a las personas en categorías, imponiéndoles una "verdad" que deben aceptar, un poder que los transforma de individuos a sujetos. Aclara que la estimulación a la belleza física (asociada a la delgadez) no solo viene de los medios de comunicación (externo) sino desde las mismas personas, en relaciones microfísicas de poder, de manera que esas normas culturales se mueven en todas direcciones $y$ en todas las relaciones sociales.

Asimismo, Visciglia (2003) realizó un estudio basado en cuatro investigaciones relacionadas al cuerpo, publicadas en la Revista de Educación Física y Ciencia, en Buenos Aires, Argentina, por la Universidad Nacional de la Plata, durante el año 2002-2003. Teoriza que 
a través del deporte se disciplinan los cuerpos; además, expresa que la actividad física y los medios de comunicación regulan la sociedad, desviando su atención para poder tener un mayor control de esta.

Oliveira, Santos y Silvestre (2012) realizaron un estudio cualitativo con 30 mujeres, las cuales tenían un promedio de edad de 46 años $y$ un promedio de 11 años de realizar actividad física. Estas mujeres fueron elegidas al azar entre seis gimnasios de Recife (Brasil). Se les realizó entrevistas y se analizaron sus discursos, encontrando que existe miedo y temor por el aumento de peso (lipofobia). Asocian estos resultados al discurso médico, los medios de comunicación, los discursos de la moda y ciertas prácticas corporales de corrección, en función de la delgadez para cumplir las expectativas sociales. Las mujeres del estudio asociaron la grasa a la enfermedad, a lo desproporcionado o lo feo y a lo improductivo. Ante esto, el equipo investigador se refiere a la fuerza de la industria de la belleza desde dos aspectos: la consideración del cuerpo inacabado y lleno de imperfecciones que pueden ser corregidas $y$ al surgimiento de una moral somática centrada en la apariencia para lograr éxito y placer. Concluyen que ha surgido una cultura lipofóbica en las sociedades occidentales, a la que se debe prestar atención.

Duncan (2007) en su ensayo cita las investigaciones de Blaine y McElroy (2002); Chambliss, Finley y Blair (2004); Schwartz, Vartanian, Nosek y Brownell (2006), quienes muestran la forma en que la sociedad etiqueta a la personas obesa como de voluntad débil, autoindulgente, perezoso, poco atractivo, torpe, estúpido, y sin valor.

Blaudeau, Hunter y Roy (2006) examinaron la relación entre capacidad aeróbica, actividad física, porcentaje de grasa corporal con las variables socioeconómicas, la percepción de la forma del cuerpo y la insatisfacción de estudiantes en edad universitaria. Participaron 153 mujeres euroamericanas, que se inscribieron en un programa de salud y clase de gimnasia en el sur de Estados Unidos. Encontraron que las participantes están insatisfechas con su cuerpo y desean estar más delgadas, a pesar de que muestran índices de masa corporal y porcentaje de grasa normal; además creen que los hombres prefieren cuerpos delgados y ellas mismas perciben estos cuerpos más atractivos. De esta manera, se evidencia que la forma corporal ideal está influenciada por lo que se encuentra atractivo.

Camacho, Rodríguez y Moreno (2002) realizaron un estudio con 690 estudiantes de educación secundaria, en cuatro centros públicos de la Comunidad Autónoma de Madrid, de los cuales 255 eran hombres y 435 mujeres con edades entre los 12 y 18 años. Midieron el grado de insatisfacción corporal con las partes del cuerpo y con el peso, las razones por las cuales deseaban cambiar el peso y las prácticas de control llevadas a cabo por los jóvenes. Se encontró que las mujeres presentan mayor insatisfacción que los hombres en cada una de las partes del cuerpo, principalmente se encuentran insatisfechas con los muslos, piernas, cadera y nalgas; los hombres se encuentran insatisfechos con la cintura y el estómago. Mientras, hombres como mujeres están insatisfechos con el peso.

Esta investigación confirma que existe una tendencia evolutiva de insatisfacción corporal, $y$ concluye que a mayor edad, mayor es la insatisfacción. También se detalla que a medida que aumenta el peso corporal, aumenta la insatisfacción corporal y esta conduce a los investigados a tener deseos de modificar su peso corporal (Camacho, Rodríguez y Moreno, 2002).

Además, se determina que las prácticas llevadas a cabo para bajar de peso por los participantes, son mayores en las mujeres. Las principales prácticas son privación de alimentos y ejercicio físico. Entre las principales actividades físicas practicadas destacan: ejercicio aeróbicos como natación, atletismo, ciclismo y actividades de gimnasia de forma, para el mantenimiento y la musculación (Camacho, Rodríguez y Moreno, 2002).

Asimismo, Macedo, Machado, Miguel, Oliveira, Perrout y Silva (2011) realizaron un estudio cuantitativo en el que midieron las dimensiones antropométricas relacionadas con la insatisfacción de la imagen corporal de 276 miembros brasileños de centros de 
acondicionamiento físico (168 hombres y 108 mujeres) con edades entre 17 y 39 años. Después de medir la masa corporal, el índice de masa corporal y la grasa corporal, las personas participantes indicaron sus siluetas actuales e ideales, permitiendo el cálculo de la insatisfacción corporal como la diferencia entre ambas, detectando una minoría de mujeres satisfechas con su cuerpo.

Carbajal, Cuadrado y Moreiras (2000) evaluaron la relación entre el índice de masa corporal y el deseo de perder peso corporal, los participantes completaron un cuestionario y se les realizaron medidas antropométricas a 40 hombres y 105 mujeres con edades entre los 12 y los 18 años de diferentes centros educativos de Madrid. Encontraron que las mujeres de su estudio tienen mayor insatisfacción corporal que los hombres, $y$ que la principal actividad desarrollada por todos los participantes para bajar de peso es la actividad física y el deporte.

Además, Sands y Wardle (2003) realizaron medidas antropométricas $y$ aplicaron un cuestionario a 356 niñas de escuelas en Londres (Inglaterra) sobre la insatisfacción corporal, la conciencia e interiorización del ideal de delgadez. Encuentran que la interiorización del ideal de delgadez es un elemento central en el desarrollo de la insatisfacción corporal, que se produce a una edad temprana en algunas chicas.

En esta misma línea, Jankauskiené y Pajaujiené (2012) analizaron la interiorización del ideal sociocultural, la insatisfacción corporal $y$ el control del peso en atletas $y$ no atletas adolescentes. Las investigadoras indican que el grupo de adolescentes investigado se preocupa por el tamaño y la forma de su cuerpo, a raíz de las presiones sociales que incitan al ideal de delgadez. Encontraron que los participantes que interiorizan las normas sociales del cuerpo estaban más insatisfechos con su apariencia. En el estudio se tomaron en cuenta 10 escuelas y 6 gimnasios seleccionados al azar, participaron de manera voluntaria 805 adolescentes, con un promedio de edad de 17-23 años, de los cuales el 58,9 \% eran mujeres (476). Todas las personas participantes completaron un cuestionario.
Oliveira (2012) realizó un estudio con 237 adolescentes de 14 a 17 años y 205 universitarios de 19 a 27 años, todos matriculados en centros públicos y universidades de la provincia de Toledo, para recolectar los datos se aplicaron cuestionarios: BSQ-34 Body Shape Questionnaire (evalúa insatisfacción corporal), сімес-26 (Cuestionario de Influencia del Modelo Estético Corporal y la Escala de Satisfacción de área corporales), así como, grupos de discusión que fueron analizados con el programa Atlas. Ti 5.

Se comprobó que la sobrevaloración del modelo estético corporal "ideal" por parte de los medios de comunicación influye negativamente en la autoevaluación de la imagen corporal, siendo las chicas quienes están más influenciadas por el modelo estético corporal de delgadez. Concluyó que a medida que aumenta la influencia de los medios de comunicación, aumenta la insatisfacción corporal y viceversa. Además, esa insatisfacción surge al comparar el yo corporal real con el yo corporal ideal. Se observa que la población estudiada recurre a prácticas de cuidado del cuerpo como actividad físico-deportiva que, en este caso, el grupo de participantes del estudio la identificaron como un factor positivo pero también como un factor de riesgo. Se concluyó que la insatisfacción corporal disminuye conforme aumenta la edad, esa insatisfacción corporal se refleja en las chicas en el deseo de perder peso y en los chicos en el deseo de ganar peso y musculatura (Oliveira, 2012).

Greenleaf, McGreer y Parham (2006) estudiaron la percepción del ideal corporal $y$ las experiencias de la imagen corporal en 5 instructores y 6 participantes de clases de aeróbicos, a los que se les realizó entrevistas. Las participantes describen el ideal corporal como delgado y tonificado pero sin tanto músculo. Las participantes de las clases tenían un promedio de edad entre 21 y 25 años, un mínimo de 2 años de participar en clases de aeróbicos, mientras que los instructores de 22 a 50 años de edad, entre 1 y 19 años de experiencia. Ellas pensaban que podían conseguir el ideal corporal. 


\section{3) METODOLOGÍA}

Para este estudio se trabajó con mujeres de la provincia de Cartago, practicantes de actividades físico-deportivas realizadas en gimnasio, específicamente del cantón central de Turrialba y de Paraíso; todas mayores de 18 $y$ menores de 60 años. Se seleccionó la muestra de manera incidental para responder a la comodidad de la investigadora, según criterios de fácil acceso, posibilidad de establecer una buena relación entre la entrevistada y la investigadora, generar credibilidad en la información, interés de la participante, comunicación y disponibilidad de tiempo para participar de las entrevistas.

La muestra se seleccionó teniendo en cuenta la edad y el tiempo que tienen de asistir al gimnasio (antigüedad). De las 14 mujeres seleccionadas, 6 son principiantes (tienen menos de tres meses de asistir al gimnasio de forma continua) y 8 son no principiantes (tienen más de tres meses de asistir al gimnasio de forma continua). De las 14 mujeres, 4 se encuentran en un rango de edad entre los 18-30 años, 5 en un rango de edad entre los 31-45 años y 5 en un rango de edad entre los 46-60 años. El promedio de edad de las entrevistadas es de 40 años.

De las entrevistadas, 5 mujeres asisten al Gimnasio Snake y 5 mujeres asisten al gimnasio Eben Ezer (ambos ubicados en el cantón central de Turrialba) y 4 mujeres asisten al gimnasio ROD-Do (ubicado en el cantón de $\mathrm{Pa}$ raíso). Los gimnasios se seleccionaron según la ubicación y los servicios que brindan. Además, se buscó que ofrezcan variedad de servicios, por lo tanto, con diferentes espacios para prácticas físicas distintas.

De ellas, 7 son casadas, 1 separada, 3 viven en unión libre y 3 son solteras; todas trabajan y son heterosexuales; la mayoría ha cursado estudios universitarios, 9 son profesionales, 3 terminaron la secundaria y 2 terminaron la primaria.

Se usó la técnica de la entrevista en profundidad. Por medio de esta se procuró una conversación dirigida hacia los temas de interés para esta investigación, la cual se focalizó de acuerdo con el tema de estudio y sus subtemas. No se estableció un número de entrevistas por participante, pues era posible que con algunas de las mujeres se necesitaran varias entrevistas para cumplir el objetivo, sin embargo, todas prefirieron que se les realizará la entrevista en un solo día. Se procuró un ambiente distendido, informal, cómodo y de confianza para la entrevistada.

La mayoría de las entrevistas se realizaron en el gimnasio o en lugares seleccionados por las entrevistadas, como la casa de habitación o lugar de trabajo. Se solicitó expresarse con sinceridad, en sus propias palabras, se les explico el interés en conocer la forma en que ven, sienten o viven. Se procuró facilitar y estimular la expresión de cada una. Se usó grabadora con previa autorización para registrar los detalles de la entrevista, con el fin de prestar la suficiente atención a la entrevistada y posibilitar la transcripción sin alteraciones.

Las entrevistas fueron individuales para que la entrevistada fuera la única protagonista, tuviera el tiempo suficiente para responder y no se sintiera intimidada porque las demás escuchen sus respuestas

El momento de la entrevista fue determinado por la entrevistada, según la disponibilidad que tuviera. Las preguntas realizadas ya estaban previamente establecidas en un instrumento que fue guía para la entrevista. Esta guía fue aplicada previamente a tres mujeres con el fin de someterla a prueba $y$ hacer mejoras, se eliminaron algunas preguntas, mejorando la redacción y agregando preguntas un poco más específicas, una vez realizadas las mejoras se aplicó a la muestra.

Los pasos seguidos en el proceso de análisis que permitieron ordenar, categorizar, priorizar e interrelacionar los datos, fueron los siguientes:

1) Realización de las entrevistas.

2) Transcripción de las entrevistas.

3) Lecturas preliminares del texto transcrito.

4) Marcar, señalar y subrayar fragmentos del texto que correspondan a cada subcategoría y categoría de análisis para seleccionarlos. Al mismo tiempo, se hicieron anotaciones sobre narraciones interesantes e importantes. 
5) Se agruparon los fragmentos de texto seleccionados por tema de análisis. En la mayoría de ellos, se cita lo que expresó la entrevistada con las mismas palabras, con el fin de no alterar la información.

6) Posteriormente, se juntaron todos los fragmentos seleccionados y las anotaciones de una misma entrevista para compararla con las de las otras entrevistas.

7) Una vez integrados todos los fragmentos se procede a interpretarlos y contrastarlos con la teoría.

Con cada una de las entrevistas se siguió los pasos anteriores. Además, se procesaron las respuestas en tablas de frecuencia con el fin de mostrar la cantidad de respuestas asignadas a un elemento analizado.

\section{4) RESULTADOS Y DISCUSIÓN}

\section{ACCIONES PARA CONTROLAR Y MOLDEAR EL CUERPO}

Las mujeres entrevistadas afirman recurrir a varias acciones para controlar y moldear su cuerpo, entre estas: la dieta, mirarse en el espejo, control del peso en la báscula, control del peso con la ropa y actividad físico-deportiva (AFD). Según la frecuencia en las respuestas, la principal acción a la que recurren es ir al gimnasio a practicar AFD.

Los resultados concuerdan parcialmente con lo obtenido por Camacho et ál. (2002) quienes encontraron que las mujeres recurren principalmente a dos acciones para controlar su cuerpo: privación de alimentos y ejercicio físico. Además, coincide con los resultados obtenidos por Carbajal et ál. (2000) quienes encontraron que la principal actividad desarrollada por las mujeres para bajar de peso es la actividad física $y$ el deporte.

Al comparar los promedios de las frecuencias de respuesta según edad y antigüedad de las mujeres entrevistadas, no se encontró mayor diferencia en cuanto al promedio de acciones a las que recurren.

Todas las entrevistadas dicen que las AFD que practican les permiten tener un cuerpo femenino. Al analizar las entrevistas de forma integral, según la frecuencia de las respuestas se determina que el significado de feminidad que ellas asumen proviene, primordialmente, de dos factores: apariencia y el comportamiento, los cuales asocian la feminidad en relación directa con el cuerpo. Sin embargo, según ellas tener un cuerpo femenino producto de la AFD tiene un costo físico, por ejemplo, destacan algunas expresiones en las que se refieren a la AFD como una "matada". Es decir, realizan un gran esfuerzo para obtener lo que se quiere, por lo que podrían interpretarse como actividades en las que se acaba con el cuerpo que se posee para construir uno diferente, un cuerpo que se adapta a un contexto y momento sociocultural, la muerte del individuo para dar paso a un sujeto corporalizado.

No obstante, las AFD no solo disciplinan los cuerpos, sino también según Gambau et ál. (2003) consolidan concepciones de género sobre los ideales de belleza y perfección. De acuerdo con estos autores, "de entre las prácticas sociales que moldean los cuerpos y modelan las ideas sobre los cuerpos, el deporte contribuye a crear y consolidar determinadas concepciones de género" (p. 127). Estos mismos autores afirman que el deporte junto con otras prácticas sociales, es un nutriente importante de los ideales de belleza y de perfección.

De esta manera, ellas disciplinan sus cuerpos por medio de la actividad física para obtener un cuerpo femenino, tal como dice Lee (1997), cuando señala que hay prácticas disciplinarias que producen $y$ construyen cuerpos femeninos con cierto tamaño, postura, movimiento, ornamenta y apariencia física. Además, agrega que "la subjetividad femenina se constituye significativamente en y a través de las prácticas disciplinarias que construyen el cuerpo femenino" (p. 146). Adicionalmente, Tubert (2010) menciona que la dieta y el ejercicio son prácticas normativas de la feminidad, lo explica de la siguiente manera:

La dieta y el ejercicio, que surgen de las prácticas normativas de la feminidad en nuestra cultura a las que reproducenpreparan al cuerpo femenino para la docilidad y la obediencia. No obstante, 
las mujeres experimentan estas prácticas como fuentes de poder y control, pues las perciben como medios para alcanzar la belleza, aceptación social, laboral y sexual (p. 166).

Estas mujeres disciplinan sus cuerpos por medio del ejercicio físico y la dieta, sometiéndose a controles usando la báscula, la ropa y el espejo; fundamentadas en la preocupación no solo de tener un cuerpo femenino, sino apariencia femenina $y$ esto aparece directamente relacionado con el peso corporal.

Por lo tanto, para ellas es importante disciplinar su cuerpo usando diferentes formas de control. Se vuelven vigilantes del proceso disciplinario del que son partícipes al estilo del esquema panóptico ${ }^{3}$, así como lo explica Bentham (2011), al exponer que este dispositivo procura que en todo momento la persona tenga razones para creerse vigilado; además, agrega que este mecanismo optimiza el control social y permite que las personas se comporten siempre como si estuvieran bajo vigilancia (Bentham, 2011).

\section{CONTROL DE LA ALIMENTACIÓN}

De las participantes, 10 se preocupan cuando comen alimentos dulces como chocolates, pasteles, postres, helados, etc. Esta preocupación que sienten refleja la presión social que existe por el cuerpo y la apariencia. Tal como lo expresa Martínez (2004), al afirmar que el cuerpo tiene presiones sociales diferentes, como los discursos de la salud y la imagen que promueven prácticas de cuido corporal; en este caso una de las prácticas a las que recurren para el cuido corporal es el control en la alimentación. Para ellas, ingerir alimentos dulces es una conducta inapropiada que va en contra de las acciones a las que recurren para disciplinar el cuerpo.

Las mujeres de este estudio clasifican sus conductas alimenticias en apropiadas o inapropiadas, buenas o malas, y penalizan las

3 El panóptico es definido como un dispositivo de vigilancia, en el que se impone una conducta mientras se mira a los otros sin ser vistos. conductas consideradas "inapropiadas". De acuerdo con Foucault (2008), esto es parte de un mecanismo disciplinario en el que se califican las conductas a partir de valoraciones opuestas y se jerarquiza a las personas (Foucault, 2008).

En este sentido, Ferrús (2007) detalla que las prácticas disciplinarias provienen del ámbito religioso, pero el deporte ha sustituido a la religión en el disciplinamiento. Baudrillard (2012) se refiere a esa sustitución afirmando que "el cuerpo hoy ha llegado a ser objeto de salvación. Ha sustituido literalmente al alma en su función moral e ideológica" (p. 155). En las entrevistas realizadas destacan los términos pecado $y$ conciencia para referirse al incumplimiento del disciplinamiento.

En este caso, las mujeres entrevistadas creen que ingerir dulces, no ir al gimnasio o no hacer AFD, es un pecado o una falta a los mandamientos sociales que las aparta de obtener la gracia de un cuerpo ideal, ser saludables, atractivas y bonitas. Esta falta les hace sentir culpa, remordimiento e inclusive arrepentimiento, experimentan una batalla entre lo que se quiere hacer $y$ ser, $y$ lo que se les exige. Ellas tienen identificadas las fuentes de pecado $y$, cuando caen en ellas, se castigan. La falta de ejercicio físico es un pecado que les causa remordimiento de conciencia, mientras que aumentar el ejercicio físico les hace sentirse bien.

Esta analogía entre los resultados obtenidos y los fundamentos del cristianismo conduce a una discusión sobre moralidad. Los mandatos de la moral que se conocen, por ejemplo, el respeto a la vida $y$ los bienes ajenos, se mantienen y se agregan otros, según la sociedad y el momento vivido; uno de los mandatos que deben cumplir las mujeres es tener un cuerpo delgado y una apariencia de cuido. Efectivamente, el cumplir esos mandatos les genera limitación para elegir y las conduce al cumplimiento de un código moral.

Al respecto, Nietzsche (1932) menciona que la moral y la religión han hecho del humano lo que han querido. Además, que dan "preceptos acerca de cómo se debe vivir y hacen que se obedezcan estos preceptos por medio de castigos y recompensas" (p. 93); establece que 
"la concepción de mundo está manchada por la idea del castigo" (p. 90).

Conjuntamente, el cuerpo no ha dejado de ser castigado, tal como ocurría en la tradición cristiana, pero está en juego la confrontación entre el cuerpo disciplinado y el cuerpo indisciplinado. Vidas, cuerpos, apariencias y cotidianidades homogéneas es lo que busca la sociedad actual, en la que las diferencias son rechazadas y discriminadas. Cuerpos saludables, "atractivos" y delgados son aceptados, mientras los cuerpos que no se ajustan a estos moldes serán sancionados.

Además, esa preocupación que ellas manifiestan se relaciona con el concepto de panóptico, el cual en la práctica da como resultado el autocontrol del cuerpo; al sentirse vigiladas y observadas por otros, caen en la auto-vigilancia de lo que se come y de la actividad física que se hace.

\section{EL CONTROL CORPORAL}

Las participantes alegan que los encargados del gimnasio controlan su cuerpo cada mes con toma de medidas antropométricas $y$ peso, afirman que se sienten satisfactoriamente si bajaron de peso, no obstante, si los resultados no son los esperados se sienten mal. Esto se relaciona con lo expuesto por Carro (2011), ella explica que "la medida se ha convertido al día de hoy en herramienta para la normalización del canon de belleza femenina; representa pues el instrumento para la internalización del sometimiento" (p. 59). Esos controles mensuales funcionan como un examen, como parte del procedimiento disciplinario que permite vigilar y sancionar; el examen es una de las claves en el éxito del poder disciplinario (Foucault, 2008).

Es notable la participación de las participantes $y$ del entrenador en el control corporal. Así, su identidad corporal y su propia subjetividad se configuran a partir de las relaciones con los otros, desde relaciones de obediencia $y$ dominación, de manera tal que el disciplinamiento del cuerpo no solo es producto de un proceso subjetivo, sino de un proceso de intersubjetividad, es decir, uno en el que intervienen otras personas.
Desde el discurso de la salud pero inspiradas en un ideal corporal, ellas entrenan, vigilan y domestican sus cuerpos, controlándose por medio de la alimentación, la actividad física, el peso y la toma de medidas físicas; lo hacen ellas mismas pero también le ceden esa potestad a la persona instructora. Este ocupa un puesto de juez del cuerpo, por ende, el entrenador o entrenadora también es parte de ese proceso intersubjetivo.

Para las mujeres entrevistadas el cuerpo ocupa un lugar protagónico en sus vidas, ese cuerpo es observado por otras personas y evaluado, hasta el punto de naturalizar esas evaluaciones en su cotidianidad.

El cuerpo se construye $y$ se produce en función de las otras personas, sobre el cuerpo recae el poder que disciplina, vigila y castiga. Además, está sujeto a una ética que establece lo permitido, lo prohibido e inclusive las normas sobre el cuidado, el entrenamiento físico y la apariencia. Por lo tanto, es por medio del cuerpo que el individuo se convierte en sujeto, lo cual ha conducido a un modo de existir en la actualidad. Sin embargo, no solo se está desarrollando un disciplinamiento del cuerpo, antes de este disciplinamiento externo se está llevando a cabo uno interno de sus emociones $y$ formas de pensar de manera que, antes de colonizar el cuerpo, se coloniza su pensamiento.

\section{INSATISFACCIÓN CORPORAL}

La totalidad de las entrevistadas mencionan disconformidad con algunas partes corporales, principalmente con el abdomen, aunque algunas también manifiestan en menor medida insatisfacción con sus brazos, piernas y cadera. Estos resultados tienen un alto grado de coincidencia con los obtenidos por Camacho et ál. (2002), quienes encontraron que las partes del cuerpo con las que las mujeres se encuentran más insatisfechas son piernas, cadera y glúteos. Camacho et ál. (2005) detallan que la insatisfacción que tienen las mujeres con las caderas muslos y glúteos está asociada a las características de la feminidad.

Los resultados concuerdan con la teoría de Vera (2001) sobre la existencia de una sobrevaloración de algunas partes del cuerpo 
(Vera, 2001), en este caso se sobreestima el abdomen. Ellas se evalúan teniendo en cuenta el estándar de belleza establecido socialmente, el cual es un abdomen plano y sin excesos de grasa por lo que, al no estar acordes con este tipo de abdomen, se deteriora su imagen corporal y eso las hace recurrir al control alimenticio y el ejercicio físico. Según Vera (2001) esa sobreestimación en algunas partes del cuerpo como estómago, nalgas, muslos y cadera afecta negativamente la imagen corporal y si no se cumple con el modelo socialmente aceptado o con los cánones establecidos, esto conduce a ciertas conductas de mitigación (Vera, 2001).

$\mathrm{Al}$ analizar las entrevistas se puede determinar que ninguna está totalmente satisfecha con su apariencia. La insatisfacción que sienten sobre sus cuerpos es denominada por algunos autores y autoras como malestar, inconformidad, insatisfacción o incomodidad. La insatisfacción corporal en las mujeres entrevistadas se debe al ideal corporal probablemente relacionado con los estereotipos de género.

En esta investigación no se encontró relación entre el peso y la insatisfacción corporal, porque indiferentemente del peso, todas las entrevistadas manifestaron insatisfacción corporal. Adicionalmente, en la muestra analizada no se encontró relación entre la insatisfacción corporal de las mujeres más jóvenes y las mayores, porque a pesar de la edad, todas están insatisfechas con su cuerpo. Pero sí se puede afirmar que las mujeres entrevistadas tienen una mala imagen corporal e insatisfacción corporal.

\section{INSATISFACCIÓN Y EL ÍNDICE DE MASA CORPORAL (IMC)}

$\mathrm{Al}$ analizar el IMC ${ }^{4}$ de las entrevistadas se determina que la insatisfacción corporal que ellas experimentan no necesariamente se debe a la composición corporal en su totalidad, sino a otros factores, como por ejemplo, a la comparación entre el cuerpo real y el ideal corporal. Al relacionar el peso y la talla, se encuentra que 6 tienen un peso normal según los parámetros de la Organización Mundial de la Salud para

4 El Índice de Masa Corporal (IMC) es la medida del peso de una persona en relación con su altura. evaluar riesgos asociados a la obesidad, pero estas 6 mujeres, al igual que las otras entrevistadas, también están insatisfechas con el cuerpo, lo cual demuestra que no existe concordancia entre la imagen corporal que estas seis mujeres tienen de su cuerpo y la apariencia real, o bien, el peso real de estas mujeres no incide en su auto-imagen corporal.

De las entrevistadas, 6 tienen un peso normal pero consideran estar "gordas", desean adelgazar y se sienten mal con su peso pues consideran que la gordura significa estar fuera de la tendencia y lo asocian al fracaso.

Por lo tanto, se difiere de los resultados obtenidos por Martínez y Veiga (2007) quienes afirman que valores más altos en el IMC se asocian con una mayor insatisfacción corporal (Martínez y Veiga, 2007). La insatisfacción que sienten las mujeres que tienen un IMC "normal" podría deberse a una distorsión de la imagen corporal, lo que originaría entre las entrevistadas una insatisfacción generalizada a pesar del IMC.

Por el contrario, los resultados si coinciden con los de Blaudeau et ál. (2006), quienes encontraron en su investigación que a pesar de que las mujeres tenían índices de masa corporal y porcentajes de grasa normal, no estaban satisfechas con su cuerpo.

\section{IDEAL DE MUJER BONITA, ATRACTIVA Y SALUDABLE}

Las acciones efectuadas por estas mujeres para moldear y controlar su cuerpo, se realizan para verse atractivas, bonitas y saludables, tal como lo menciona Vázquez (2000), en la sociedad actual se otorga relevancia al cuerpo por la centralidad de tres aspectos: belleza, salud y rendimiento (Vázquez, 2000). A la pregunta, ¿cómo debe verse una mujer bonita, atractiva $y$ saludable?, 8 mujeres respondieron que debe tener bajo peso y 6 dijeron que debe tener una buena presentación personal; otras respuestas fueron tener un cuerpo tonificado, verse limpia, coqueta y agradable. Estos son elementos valorativos que utilizan para determinar a una mujer bonita, atractiva y saludable.

Cuando se les pregunta qué se debe hacer para verse bonita, atractiva $y$ saludable, 12 mencionan hacer ejercicio, otras respuestas 
fueron cuidar la alimentación, maquillarse, cuidar la apariencia y usar ropa apropiada, juvenil $y$ ajustada.

Según las entrevistadas, para que una mujer tenga salud, sea bonita $y$ atractiva no debe ser gorda, ya que relacionan a una persona "gorda" con alguien que no está bien físicamente y que está enferma, mientras que una persona que no tiene gordura, para ellas es saludable, atractiva $y$ bonita. Lo anterior coincide con lo que expresa Duncan (2007) sobre los estereotipos que existen hacia las personas con sobrepeso, las cuales son consideradas poco atractivas. Además, los resultados concuerdan con los obtenidos por Oliveira et ál. (2012) quienes encontraron que las mujeres asocian la grasa con la enfermedad $y$ lo feo.

En algunas ocasiones, la actividad física funciona como un castigo por algún alimento dulce que comieron, ellas mismas se castigan duplicando la actividad física. ¿Qué es tan importante para que una mujer vigile lo que consume y cómo lo gasta? Ellas tienen miedo y temor de aumentar de peso. Oliviera et ál. (2012), lo llaman "lipofobia" y lo asocian con el discurso médico, los medios de comunicación, discursos de la moda y ciertas prácticas corporales de corrección, en función de la delgadez para cumplir las expectativas sociales.

Se refieren a la fuerza de la industria de la belleza desde dos aspectos: la consideración del cuerpo inacabado y lleno de imperfecciones que puede ser corregido $y$ al surgimiento de una moral somática centrada en la apariencia para lograr éxito y placer (Oliviera et ál., 2012). Por otra parte, Gracia (2014) también se refiere a la lipofobia y la asocia a los hábitos alimenticios y a la enfermedad, de manera que las personas no quieren engordar porque evidenciarían glotonería y problemas en su salud.

Cuando se les consulta sobre por qué es importante para ellas verse bonitas, atractivas $y$ saludables, mencionan que para sentirse bien, para ser aceptadas, por el trabajo que tienen, para ser ejemplo para sus hijas $y$ por el esposo. El tener sobrepeso sería un mal ejemplo para sus hijas, podrían no ser aceptadas y tendrían problemas en sus trabajos. Por lo tanto, ellas modifican sus cuerpos por aceptación y aprobación de los otros; esto determina la forma en que ellas habitan sus cuerpos, cuerpos en los que es muy importante lo ornamental, la postura $y$-principalmenteel volumen del cuerpo.

\section{5) CONCLUSIONES}

Se demuestra que las entrevistadas recurren a varias acciones para controlar y moldear el cuerpo, entre las que se encuentran la actividad física, la dieta, el control del peso en la báscula, el control con la talla de la ropa y el control en el espejo. Asimismo, la AFD es la principal acción a la que recurren para bajar de peso y tener salud, esto coincide con Visciglia (2003) quien afirma que por medio del deporte se disciplinan los cuerpos.

No se encontró diferencia entre la cantidad de acciones que realizan las entrevistadas según edad $y$ antigüedad o tiempo que tienen de hacer AFD, esto al comparar los promedios de las frecuencias de respuesta.

Existe consonancia con Camacho et ál. (2002), quienes también encontraron que la AFD y la dieta son las principales acciones para controlar el cuerpo, y con Carbajal et ál. (2000) al encontrar que la principal acción a la que recurren para controlar el cuerpo es la AFD.

Se comprueba que ellas recurren a las prácticas disciplinarias para controlar y moldear sus cuerpos, así como para construir cuerpos femeninos (Tubert, 2010; Gambau et ál., 2003 y Lee, 1997). Se concluye que el disciplinamiento es un proceso intersubjetivo, desarrollado desde el autocontrol, dando protagonismo al cuerpo, que es moldeado según el ideal de cuerpo existente acorde a los estereotipos de género.

El disciplinamiento corporal se desarrolla desde relaciones microfísicas de poder a las que se les debe prestar detenida atención, es el caso de la relación entre madre e hija, trabajadora $y$ empleador(a), instructor y cliente.

Este proceso investigativo permite vislumbrar que existen ideas actuales que circulan en la sociedad que se contraponen a la naturaleza del cuerpo de las mujeres, como lo son las etiquetas negativas asignadas a la grasa y el aumento de peso. Finalmente, las mujeres entrevistadas a raíz de la insatisfacción corporal 
recurren a acciones para moldear, controlar y modificar el cuerpo con el fin de transformarlo y mejorarlo, hasta obtener cuerpos "ideales", desde un autocontrol consiente ejercido sobre el cuerpo.

\section{REFERENCIAS}

Andrews, D., Cole, C., y Giardina, M. (2004). Michael Foucault: studies of power and sport. Por Giulianotti, R. En: Sport and modern social theorist. New York: Palgrave Macmillan

Batres, C., Krones, P., Stice, E., y Orjada, K. (2005). In vivo social comparison to a thin-ideal peer promotes body dissatisfaction: a randomized experiment. The International Journal of Eating Disorders, 38(2), 134-142.

Baudrillard, R. J. (2012). La sociedad de consumo: sus mitos, sus estructuras. Madrid: Ediciones siglo XXI.

Bautista, R. J. (2009). El laberinto de la feminidad y el acto analítico. Buenos Aires: Homo Sapiens.

Bentham, J. (2011). Panóptico. Madrid: Ediciones Utopía. Traducción de David Cruz Acevedo.

Bordo, S. (1992). The Body and the reproduction of femininity: a feminist appropriation of Foucault. En Gender Body Knowledge, Feminist Reconstructions of Being and Knowing. New Jersey: Rutgers University Press.

Bourdieu, P. (2000). La dominación masculina. Barcelona: Editoral Anagrama.

Butler, J. (2007). El género en disputa: el feminismo y la subversión de la identidad. Barcelona: Paidós

Blaudeau, T., Hunter, G., y Roy, J. (2006). Percent body fat is related to bodyshape perception and dissatisfaction in students attending an all women's college. Perceptual and Motor Skills, 103, 677-684.

Camacho, M.M,; Rodríguez, G.M. y Moreno, D.A. (2002). La insatisfacción corporal de las adolescentes y la práctica de ejercicio físico como método de control de peso corporal. Mujeres y actividades físico deportivas, Serie número 35. Madrid: Consejo Superior de Deportes.

Camacho. M.M. (2005). Imagen corporal y práctica de actividad físico deportiva en la adolescencia (Tesis Doctoral). Universidad Complutense de Madrid, Madrid.

Caamaño, M.C; y Rangel, N. A. (2002). Maternidad, feminidad y muerte. San José: Editorial Universidad de Costa Rica.

Carbajal, A; Cuadrado, C y Moreiras, O. (Marzo, 2000). Body perceptions and slimming attitudes reported by Spanish adolescents. European Journal of Clinical Nutrition, 54, S65-S68.

Carro, F.S. (2011). Deconstruyendo la belleza. En In corpore dominae, cuerpos escritos/cuerpos proscritos. Sevilla: ArCibel Editores.

Castro, L. N. (2012). Imagen corporal, adolescencia, educación física y actividades físico-deportivas: diseño y validación de un instrumento para su análisis (Tesis doctoral). Universidad Castilla La Mancha, España.

Cerquera, C. A y Bibiana, V. C. (2012). Identificación de los estereotipos sobre envejecimiento femenino, presentes en un grupo de mujeres jóvenes. Pensamiento Psicológico, 10(1), 77-88.

Duncan, M. (2007). Bodies in Motion: The Sociology of Physical Activity. American Academy of Kinesiology and Physical Education, 59, 55-66.

Ferrús, B. (2007). Masculino y femenino en los tiempos del Cyborg. En Torras, F.M. (Ed). Cuerpo e identidad: estudios de género $y$ sexualidad 1. Barcelona: Edicions UAB.

Foucault, M.P. (2008). Vigilar y castigar: nacimiento de la prisión. Buenos Aires: Siglo xxI Editores.

Foucault, M. P. (1979). Microfísica del poder. Madrid: Ediciones La Piqueta.

Gambau, P.V.; Sánchez, M. R; Mosquera, G. M; y Pujadas, M. X. (2003). Deporte y postmodernidad. VII Congreso de la AEI$S A D$. Madrid: Librerías deportivas Esteban Sanz, S.L.

Gatens, M. (2002). El poder, los cuerpos y la diferencia. Desestabilizar la teoría. México: PUEG. 
González, CH. M. (1999). Hombres y mujeres. Subjetividad, salud y género. Madrid: Las palmas de Gran Canaria.

Gracia, A. M. (Marzo, 2014). De la lipofobia al lipofobismo: imágenes y experiencias en torno a la obesidad. Revista Salud (i) Ciencia, 20, 382-388.

Greenleaf, Ch.; McGreer, R y Parham, H. (Febrero, 2006). Physique Attitudes and Self-Presentational Concerns: Exploratory Interviews with Female Group Aerobic Exercisers and Instructors. Sex Roles, 54(3/4), 189-199.

Jankauskiené, R. y Pajaujiené, S. (2012). Sociocultural ideal internalization, body dissatisfaction and weight control behavior among adolescent athletes and non-athlete adolescents. Does that need education? Education Physical Training Sport, 2(85), 40-47.

Lagarde, R. M. (1996). Cuadernos inacabados: género y feminismo. Desarrollo humano y democracia. Madrid: Horas.

Lee, S. (1997). Foucault, femininity and the modernization of patriarchal power. En Conboy, K; Medina, N y Stanbury, S. (Eds.), Writing on the body female embodiment and feminista theory. New York: Columbia University Press.

Lengyel, C., Marshall, C. y Sus, A. (2012). Body dissatisfaction among middle-age and older women. Canadian Journal of Dietetic Practice and Research, 73(2), 241-247.

Macedo, J., Machado, V; Miguel, H; Oliveira, V; Perrout, J; y Silva, J. (Junio, 2011). Relationship between anthropometric variables and body image dissatisfaction among fitness center users. Revista De Psicología Del Deporte, 20 (2), 367-382.

Martínez, B.I. (2001). Género, desarrollo psicosocial y trastornos de la imagen corporal. Madrid: Instituto de la Mujer.

Martínez, B.I. (2004). Construcción psicosocial del cuerpo: implicaciones del sistema sexo-género. Seminario Permanente Mujer y deporte: Los modelos corporales dominantes, el ejercicio físico y la salud de las mujeres adolecentes y jóvenes.
Memoria de las jornadas. Universidad Politécnica de Madrid, Facultad de Ciencias de la Actividad Física y el Deporte, INEF.

Martínez, G.D; y Veiga, N.L. (Setiembre, 2007). Insatisfacción corporal en adolescentes: relaciones con la actividad física e índice de masa corporal. Revista Internacional de Medicina y Ciencias de la Actividad Física y el Deporte, 7 (27), 253-265.

Nietzsche, F. (1932). La voluntad de dominio. Ensayo de una transmutación de todos los valores. Tomo VIII. Madrid: M. Aguilar Editor.

Oliveira, I; Santos, F; y Silvestre, C. (Julio, 2012). A lipofobia nos discursos de mulheres praticantes de exercício físico. Motriz, Rio Claro, 18(3), 590-601.

Pérez, R.S. y Romero, J.G. (Marzo-Abril, 2010). Imagen corporal en mujeres de tres zonas rurales de México: percepción y deseo. Revista salud pública de México, 52(2), 111-118.

Pruis, T; y Janowsky, J. (2010). Assessment of body image in younger and older women. The Journal of General Psychology, 137(3), 225-238.

Sands, E; y Wardle, J. (2003). Internalization of ideal body shapes in 9-12 year old girls. International Journal of Eating Disorders, 33(2), 193-204.

Sossa, A. (2011). Análisis desde Michel Foucault referentes al cuerpo, la belleza física y el consumo. Revista Latinoamericana POLIS, 28. Recuperado de http://polis. revues.org/1417.

Tubert, S. (2010). Los ideales culturales de la feminidad $y$ sus efectos sobre el cuerpo de las mujeres. Quaderns de Psicología, 12(2), 161-174. Recuperao de http:// www.quadernsdepsicologia.cat/article/ view/760

Vázquez, B. (2000). La centralidad del cuerpo en la sociedad actual. Sociedad actual ¿sociedad del cuerpo? Trabajo presentado en el Seminario permanente "Mujer $y$ deporte". Memoria de las jornadas Cuerpo, Género y sociedad. Universidad Politécnica de Madrid, Instituto Nacional de Educación Física (INEF), España. 
Vera, C. (2001). Género, cultura e imagen del cuerpo. En Derecho de las mujeres a su imagen: los trastornos del comportamiento alimentario (anorexia y bulimia). Universidad de Alicante.

Visciglia, B.S. (2003). Un Cuerpo regulado, disciplinado, construido y valorizado. Universidad Nacional de la Plata.
Recuperado de http://sedici.unlp.edu. ar/bitstream/handle/10915/16451/ Documento_completo.pdf?sequence $=1$

Fecha de ingreso: 03/05/2018 Fecha de aprobación: 17/01/2019 